\title{
Effects of a programme of vigorous physical activity during secondary school physical education on academic performance, fitness, cognition, mental health and the brain of adolescents (Fit to Study): study protocol for a cluster- randomised trial
}

T. M. Wassenaar' ${ }^{1}$ C. M. Wheatley', N. Beale², P. Salvan', A. Meaney², J. B. Possee², K. E. Atherton', J. L. Duda ${ }^{3}$, H. Dawes ${ }^{2}$ and H. Johansen-Berg ${ }^{1 *}$ (i)

\begin{abstract}
Background: Early adolescence is a period of dynamic neurobiological change. Converging lines of research suggest that regular physical activity (PA) and improved aerobic fitness have the potential to stimulate positive brain changes, improve cognitive function and boost academic attainment in this age group, but high-quality studies are needed to substantiate these findings. The primary aim of the Fit to Study trial is to investigate whether short infusions of vigorous PA (VPA) delivered during secondary school physical education (PE) can improve attainment in maths, as described in a protocol published by NatCen Social Research. The present protocol concerns the trial's secondary outcome measures, which are variables thought to moderate or mediate the relationship between PA and attainment, including the effect of the intervention on cardiorespiratory fitness, cognitive performance, mental health and brain structure and function.
\end{abstract}

Method: The Fit to Study project is a cluster-randomised controlled trial that includes Year 8 pupils (aged 12-13) from secondary state schools in South/Mid-England. Schools were randomised into an intervention condition in which PE teachers delivered an additional 10 min of VPA per PE lesson for one academic year, or a 'PE as usual' control condition. Intervention and control groups were stratified according to whether schools were single-sex or co-educational. Assessments take place at baseline (end of Year 7, aged 11-12) and after 12 months (Year 8). Secondary outcomes are cardiorespiratory fitness, objective PA during PE, cognitive performance and mental health. The study also includes exploratory measures of daytime sleepiness, attitudes towards daily PA and PE enjoyment. A sub-set of pupils from a sub-set of schools will also take part in a brain imaging sub-study, which is embedded in the trial.

(Continued on next page)

\footnotetext{
* Correspondence: heidi.johansen-berg@ndcn.ox.ac.uk

${ }^{1}$ Wellcome Centre for Integrative Neuroimaging, FMRIB Centre, Nuffield Department of Clinical Neurosciences, University of Oxford, John Radcliffe Hospital, Headley Way, Oxford OX3 9DU, UK

Full list of author information is available at the end of the article
}

(c) The Author(s). 2019 Open Access This article is distributed under the terms of the Creative Commons Attribution 4.0 International License (http://creativecommons.org/licenses/by/4.0/), which permits unrestricted use, distribution, and reproduction in any medium, provided you give appropriate credit to the original author(s) and the source, provide a link to the Creative Commons license, and indicate if changes were made. The Creative Commons Public Domain Dedication waiver (http://creativecommons.org/publicdomain/zero/1.0/) applies to the data made available in this article, unless otherwise stated. 
(Continued from previous page)

Discussion: The Fit to Study trial could advance our understanding of the complex relationships between PA and aerobic fitness, the brain, cognitive performance, mental health and academic attainment during adolescence. Further, it will add to our understanding of whether school PE is an effective setting to increase VPA and fitness, which could inform future PA interventions and education policy.

Trial registration: ClinicalTrials.gov, NCT03286725. Retrospectively registered on 18 September 2017. ClinicalTrials.gov, NCT03593863. Retrospectively registered on 19 July 2018.

Keywords: Cluster-randomised trial, Adolescence, Exercise, Physical activity, Fitness, Academic achievement, Cognitive functions, Mental health, MRI

\section{Background}

Early adolescence is a period of dynamic neurobiological and psychological change [1] and provides a foundation for future health [2]. Understanding how health and education policies might steer the developmental course in a positive direction is therefore important [3].

Converging lines of research suggest that physical activity (PA) and aerobic exercise have the potential to stimulate positive brain changes, improve cognitive function and boost academic attainment in this age group. Neuroscience studies using animal models have indicated that, at the molecular level, aerobic exercise increases levels of growth factors responsible for synaptic plasticity, particularly in the hippocampus $[4,5]$. At the cellular level, increased growth factor production is thought to promote the development of new blood vessels and neurons and their integration into existing networks of cells in this region [6]. Neuroimaging studies in adults [7], and increasingly in children $[8,9]$, have found support for PA and fitness-related changes in brain function and structure. For instance, hippocampal volume is larger in higher fit children and may mediate the relationship between fitness level and memory outcomes [10].

Meanwhile, intervention studies in schools have found some evidence that programmes of aerobic exercise can lead to improved cognitive performance, particularly in the domains of attention and executive functions (working memory, cognitive flexibility, and inhibitory control) [11-17]. Separately, there are associations between objectively measured PA and academic attainment among adolescents [18] and indications that school-based exercise interventions can improve educational outcomes in this age group $[9,19]$. The balance of evidence among adolescents favours positive associations between aerobic exercise, fitness, cognition and attainment [20], although the variety of study designs, including different types, intensities and durations of PA, makes firm conclusions difficult to draw.

The benefits of PA for physical and mental health are well known [21, 22], yet PA levels among young adolescents are low [23] and continue to decline during early adolescence [24]. Adolescence is also a peak time for the onset of mental ill-being, including vulnerability to low self-esteem $[25,26]$. There is some evidence that physical health and mental well-being and ill-being are also linked with academic attainment [27, 28], but whether these factors act as mediators in the relationship between PA and academic attainment is currently unclear.

School is a recommended setting for promoting PA among adolescents, including those from lower socio-economic backgrounds, because they spend a large proportion of their time at school and interventions can be delivered without relying on support from families [29]. There is evidence that physical education (PE) interventions to increase activity are an effective method of improving cognitive function and academic performance $[11,19]$. However, less than $50 \%$ of a typical PE lesson is spent in moderate-to-vigorous PA (MVPA), the type of activities that noticeably accelerate the heart rate [30, 31]. Short bouts of high-intensity or vigorous physical activity (VPA), during which individuals work at around $80 \%$ of their maximum heart rate for between $45 \mathrm{~s}$ and $4 \mathrm{~min}$, have been shown to deliver equivalent fitness benefits to longer, lower-intensity workouts $[32,33]$. This type of vigorous activity is brief enough to be incorporated into PE lessons without disrupting curriculum delivery, and there is evidence that it can improve adolescent fitness [34, 35].

The primary aim of the trial is to investigate the impact of a 1-year VPA intervention (Fit to Study) delivered during PE on maths attainment. To date there is little school-based evidence to test whether relationships between PA and academic achievement among young adolescents are mediated or moderated by fitness, cognitive function, mental health outcomes and/or the brain. The secondary aim of this trial is therefore to investigate whether the intervention improves fitness, cognitive performance and mental health outcomes, and changes brain structure and function, compared to usual PE lessons delivered over a school year, and to further explore relationships between these measures.

\section{Hypotheses}

The primary hypothesis for the trial is: 
1. A programme of VPA, delivered during PE lessons for one academic year (10 months), will improve maths attainment at the end of the intervention in the intervention group relative to the control group.

The secondary hypotheses are:

2. The intervention will increase VPA during PE, and fitness levels, of pupils in the intervention group relative to the control group.

3. The intervention will improve cognitive performance (processing speed, memory and executive functions) in the intervention group relative to the control group.

4. The intervention will improve physical and mental health outcomes in the intervention group relative to the control group.

5. The intervention will change brain structure and function in the intervention group relative to the control group.

\section{Methods and analysis Design}

The study is a parallel group, superiority cluster-randomised efficacy trial of a one academic year (10-month) VPA intervention versus control. Clusters are secondary state schools in South/Mid-England; participants are Year 8 pupils within those schools. The intervention is incorporated into PE lessons to minimally disturb the curriculum and provide a scalable intervention that could be easily implemented in regular school PE lessons. Trial data are collected at baseline (end of Year 7, pupils aged 11-12) and after 12 months (end of Year 8, pupils aged 12-13). Figure 1 presents an overview of the schedule for enrolment, the intervention and the assessments, according to the Standard Protocol Items: Recommendations for Interventional Trials (SPIRIT) guidelines (Additional file 1).

A sub-set of pupils from a sub-set of schools will also take part in a brain imaging sub-study which is embedded in the trial. Data for the brain imaging sub-study are collected at baseline (end of Year 7, pupils aged 11-12), after 12 months (end of Year 8, pupils aged 12-13) and at 24 months (end of Year 9, pupils aged 13-14).

The trial has been approved by the Central University Research Ethics Committee of Oxford University (Registration No. R48879). The main trial protocol was retrospectively registered at ClinicalTrials.gov on 18 September 2017 (NCT03286725). At this time, study enrolment was complete and data acquisition was in progress. The brain imaging sub-study protocol was retrospectively registered on 19 July 2018 (NCT03593863). At this time study enrolment and data acquisition were both still in progress. The primary measure, attainment in maths, is recorded separately on the ISRCTN registry (15730512).

\section{Independent evaluation}

The trial will be independently evaluated by NatCen Social Research. NatCen performed the randomisation and the trial's power calculation, and will carry out the primary intention-to-treat analysis, comparing maths performance between intervention and control schools. The protocol and statistical analysis plan for the independent evaluation has been published separately $[36,37]$.

\section{Sample selection and recruitment}

A total of 106 schools have been recruited and assessed for eligibility (Fig. 2). To be eligible, schools must (1) be secondary state or academy schools, but not grammar schools; (2) be mixed or single-gender; (3) have a proportion of pupils eligible for free school meals (FSM), preferably more than 15\%, which is the average for England (at the time of recruitment [36]); (4) be located within a pre-defined set of local authorities, encompassing the following geographical locations: Greater London; Thames Valley; Southampton and Portsmouth; Bristol and Bath; Birmingham and Coventry; Cheltenham/Gloucester; Luton, Bedford Milton Keynes; (5) have Year 7 pupils who will move on to Year 8 at the start of the intervention; (6) deliver PE as part of their curriculum; (7) sign an agreement to send opt-out consent forms to parents/carers of Year 7 pupils and inform the research team of pupils who have opted out of data storage. Following the eligibility assessment, two schools declined to participate. Of the remaining 104 schools, all pupils in Year 8 at the start of the intervention (academic year 2017-2018) are part of the trial, given that project activities are classed as part of regular school activities. However, pupils who opted out of data storage are not required to participate in any of the trial's assessments, unless decided otherwise by the PE teacher.

The National Foundation for Educational Research (NFER) led recruitment, supported by Oxford Brookes University. The NFER initially sent recruitment materials to 1125 schools, and, to improve response numbers, widened their search area to include another 223 schools. Recruitment presentations were given at County PE Teacher Conferences organised by the Oxfordshire Sports Partnership and the Buckinghamshire and Milton Keynes Sport and Activity Partnership. Those schools that wished to participate and met the inclusion criteria provided the names, dates of birth, genders, FSM status and unique pupil numbers (UPNs) of all Year 7 pupils. Schools were considered formally recruited upon transfer of the data to the NFER.

\section{Randomisation}

Schools $(n=104)$ were randomised into an intervention group or control 'PE as usual' control group using stratified 


\begin{tabular}{|c|c|c|c|c|c|c|}
\hline & \multirow[b]{2}{*}{$\begin{array}{c}\text { School / } \\
\text { individual } \\
\text { level }\end{array}$} & \multirow[b]{2}{*}{$\begin{array}{l}\text { All pupils } \\
\text { / subset }\end{array}$} & \multicolumn{4}{|c|}{ STUDY PERIOD } \\
\hline \multirow{2}{*}{ Activity or assessment } & & & $\begin{array}{l}\text { Admission to } \\
\text { trial / pre- } \\
\text { study }\end{array}$ & $\begin{array}{l}\text { Baseline/ } \\
\text { Allocation }\end{array}$ & $\begin{array}{c}\text { Post- } \\
\text { intervention }\end{array}$ & $\begin{array}{l}\text { 12-month } \\
\text { follow-up }\end{array}$ \\
\hline & & & $-t_{1}$ & 0 & $t_{1}$ & $t_{2}$ \\
\hline Eligibility screen & School & All & $\mathrm{x}$ & & & \\
\hline $\begin{array}{l}\text { Memorandum of } \\
\text { understanding of }\end{array}$ & School & All & $\mathrm{x}$ & & & \\
\hline Opt-out consent form & Individual & All & $\mathrm{x}$ & & & \\
\hline Randomisation & School & All & & $\mathrm{x}$ & & \\
\hline \multirow{3}{*}{$\begin{array}{r}\text { INTERVENTIONS: } \\
\text { Intervention: } \\
\text { additional 10min VPA per } P E \\
\text { lesson } \\
\text { Control: } \\
P E \text { as usual } \\
\end{array}$} & & & & & & \\
\hline & School & All & & & & \\
\hline & School & All & & & & \\
\hline \multirow{2}{*}{\multicolumn{7}{|c|}{ ASSESSMENTS: }} \\
\hline & & & & & & \\
\hline Physical activity during PE & $\begin{array}{l}\text { School PE } \\
\text { class }\end{array}$ & All & & $x$ & $\mathrm{x}$ & \\
\hline \multirow{2}{*}{$\begin{array}{l}\text { Physical activity during PE } \\
\text { Cognitive assessments }\end{array}$} & Individual & Sub-set & & & $\mathrm{x}$ & \\
\hline & & & & & & \\
\hline Simple reaction time task & Individual & All & & $\mathrm{x}$ & $\mathrm{x}$ & \\
\hline \multirow{2}{*}{$\begin{array}{l}\text { Modified flanker task } \\
\text { Visual 2-back task }\end{array}$} & Individual & All & & $\mathrm{x}$ & $\mathrm{x}$ & \\
\hline & Individual & All & & $\mathrm{x}$ & $\mathrm{x}$ & \\
\hline \multirow{2}{*}{$\begin{array}{r}\text { Colour-shape switch task } \\
\text { Relational memory task }\end{array}$} & Individual & All & & $\mathrm{x}$ & $\mathrm{x}$ & \\
\hline & Individual & All & & $\mathrm{x}$ & $\mathrm{x}$ & \\
\hline \multicolumn{7}{|l|}{ Psychosocial measures } \\
\hline \multicolumn{7}{|l|}{ Mental health (SDQ) } \\
\hline Self-esteem (P-SDQ) & Individual & All & & $\mathrm{x}$ & $\mathrm{x}$ & \\
\hline Sleepiness (PDSS) & Individual & All & & $\mathrm{x}$ & $\mathrm{x}$ & \\
\hline Global health (HBSC) & Individual & All & & $\mathrm{x}$ & $\mathrm{x}$ & \\
\hline \multirow{3}{*}{$\begin{array}{r}\text { PE enjoyment } \\
\text { Physical activity (HBSC) } \\
\text { Attitudes/beliefs about physical } \\
\text { activity }\end{array}$} & Individual & All & & $\mathrm{x}$ & $\mathrm{x}$ & \\
\hline & Individual & All & & $x$ & $\mathrm{x}$ & \\
\hline & Individual & All & & $x$ & $x$ & \\
\hline \multirow{4}{*}{$\begin{array}{l}\text { Fitness } \\
20 m \text { Shuttle Run or } 12 \mathrm{~min} \\
\text { Cooper run } \\
\text { Brain imaging sub-study } \\
\text { (MRI, cognition, mental \& } \\
\text { physical health, fitness, PA, gait } \\
\text { assessments }\end{array}$} & & & & & & \\
\hline & Individual & All & & $\mathrm{x}$ & $\mathrm{x}$ & \\
\hline & & & & & & \\
\hline & Individual & Sub-set & & $\mathrm{x}$ & $\mathrm{x}$ & $\mathrm{x}$ \\
\hline
\end{tabular}

Fig. 1 Fit to Study trial schedule of forms and procedures (adapted from SPIRIT figure). Abbreviations: HBSC Health Behaviour in School-aged Children, PDSS Paediatric Daytime Sleepiness Scale, PE physical education, P-SDQ Physical Self-Description Questionnaire, SDQ Strengths and Difficulties Questionnaire, PA physical activity, VPA vigorous physical activity

block randomisation and an allocation ratio of 1:1 (Fig. 2). Stratification was arranged by single-sex/co-educational status of schools. NatCen performed the randomisation using a random number generator in STATA (v.12) $[36,37]$. To reduce the risk of bias, schools were informed of their assigned group by the research team after completing baseline assessments.

\section{Intervention}

The intervention consisted of a one academic year (10 months: September 2017-June 2018) PA programme delivered by PE teachers during regular Year 8 PE lessons. In the summer term prior to the trial, Year 8 PE staff from all intervention schools were invited to attend a 2 -h face-to-face training session delivered by a team from
Oxford Brookes University who designed the intervention. Those unable to attend were asked to join an interactive 2-h online training session delivering the same content. Teachers unable to take part during the summer term attended training at the start of the autumn term. During these sessions, the intervention was described to the teachers.

The intervention requires teachers to deliver specific elements of additional VPA, over and above normal VPA, during all Year 8 PE lessons, for the whole school year. Four minutes of VPA are incorporated into the PE lesson as part of a warm-up, and three 2-min infusions per hour of PE are incorporated into the main PE class. In this study, VPA is defined as activities that raise the heart rate (HR) to $71-85 \%$ of the maximum HR (HRmax), whereas 


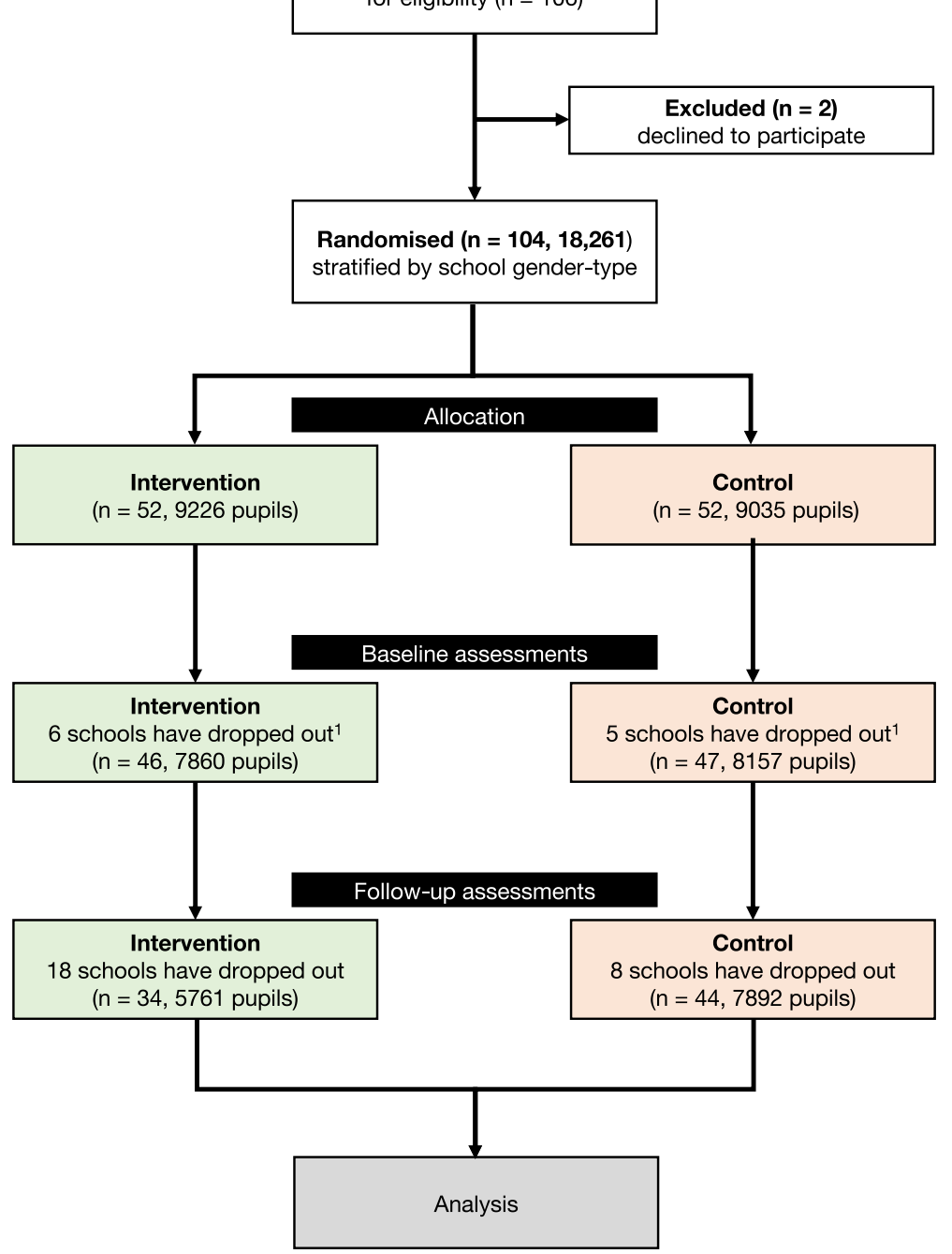

Fig. 2 Flow diagram of schools and participants. ${ }^{1}$ A total of 104 schools were randomised into an intervention and a control group. Prior to baseline assessments, 11 schools dropped out. Schools were informed of their assigned group by the research team after completing baseline assessments

moderate PA raises it to $60-70 \%$ of HRmax. The intervention elements are designed to be easy to incorporate into PE lessons, to maximise the training effect and to minimise the risk of injury with a warm-up that progresses from light to moderate-intensity activities and includes bursts of VPA.

Teachers were asked to deliver an active 10-min warm-up at the start of each lesson, beginning with light-intensity movements (e.g. wrist rotations; leg swings) and moving through moderate-intensity activity (e.g. arm rotations; walking with high knee-raise) to incorporate two periods of VPA (e.g. vigorous arm sprints; running on the spot) totalling $4 \mathrm{~min}$. Fifteen young adolescents piloted the warm-up and the infusions while wearing chest strap Polar heart rate monitors, which indicated that heart rates were in the vigorous zone for the specified time during all the intervention elements.

In addition, three 2-min infusions of VPA would be incorporated into the main PE lesson, assuming a 1-h lesson duration. If lessons were shorter or longer than 1 $\mathrm{h}$, then the number of infusions was scaled up or down accordingly. Teachers were provided with three example infusions: infusion 1 consisted of fast arm rotations (the aim is to build the intensity by speeding up arm movements concluding with two maximum effort sprints for counts of ten); infusion 2 comprised squats and lunges with an emphasis on technique and a full range of motion (to work the muscles to the maximum, concluding 
with a maximum effort sprint on the spot for a count of ten); and infusion 3 was sprinting on the spot (this must be completed at a vigorous intensity with the two 'on the spot' sprint sessions lasting for counts of ten), with active recovery periods where necessary during each 2-min session. Teachers were also invited to create their own warm-up and/or infusions to provide similar levels of VPA to fit their lesson requirements. Researchers explained the rationale for the intervention and discussed implementation strategies.

Teachers were given access to video demonstrations of the warm-up and infusion material on the trial's website. Heads of $\mathrm{PE}$ at intervention schools received emails reminding them to deliver the intervention in September and December 2017 and in February 2018. Researchers visited 30 intervention schools between September and December 2017 to answer questions and offer support and advice. PE staff were invited to join an online intervention discussion forum, and schools were offered the chance to have intervention lessons filmed. Researchers were also available to answer email and phone queries. Control schools were not given training and were asked to deliver PE as usual. During the intervention period, heads of PE in these schools received emails in September 2017 and February 2018 with information about testing. School participation was incentivised to minimise drop-out and ensure compliance. Both intervention and control schools receive $£ 500$ upon completion of the trial, following the academic attainment assessment.

\section{Intervention fidelity}

All Year 8 PE teachers in intervention schools were asked to keep a written log of VPA achieved in each PE lesson. Specifically, they were asked to record whether the class completed 4 min of VPA during the warm-up and to mark how many infusions were delivered. Teachers were informed that for a teacher-created warm-up to be marked as completed, it must have lasted $10 \mathrm{~min}$ and progressed from light-intensity small movement activities to larger moderate-intensity activities with short bursts of vigorous-intensity activities. For a teacher-created infusion to be marked as completed, it must have lasted $2 \mathrm{~min}$ and included two short bursts of VPA (or equivalent, as per infusion 2, described in the 'Intervention' section).

Further, researchers are visiting a convenience sample of intervention and control schools during 2018 to take objective measurements of PA during PE using Axivity AX3 accelerometers (see 'Physical activity during PE' in the subsequent section 'Secondary outcome measures'). Finally, a subjective measure of compliance will be collected from participants from all schools during post-intervention testing using a brief three-item survey asking whether (1) PE lessons start with a warm-up, (2) PE lessons include bursts of VPA that raise their heart rate and make them feel out of breath and (3) participants take part in warmups or VPA bursts if asked by PE teachers.

\section{Blinding}

Researchers assessing secondary outcomes are blinded to intervention/control status of schools, but those who are visiting schools to collect fidelity measures and provide top-up intervention training are not blinded. Schools were informed of their status following baseline measurements, so that intervention school teachers could receive training and deliver the intervention.

\section{Outcome measures}

\section{Primary outcome measure}

The primary outcome measure is academic attainment, assessed by the Progress Test in Maths (PTM), Level 13 [38]. To reduce the test burden, NatCen will administer the test to a random selection of half of all form groups per school, post-intervention only [37].

\section{Secondary outcome measures}

Cardiorespiratory fitness Fitness is being assessed using the 20-m multistage shuttle run test [39]. This well-validated test [40] measures participants' maximum aerobic capacity. Schools with a policy of not using the multistage fitness test are completing the Cooper Run Test [41], which requires pupils to run or walk as far as possible in $12 \mathrm{~min}$ around a measured track (between 80 and $400 \mathrm{~m}$ ). Fitness measures are being collected once at baseline and once during the summer term. The tests are conducted by PE teachers during regular PE lessons to minimise the burden on participants and to avoid disrupting other classes.

Cognitive and mental health assessments Cognitive and mental health measures are being collected once at baseline and once during the summer term at the end of the intervention. All cognitive assessments and health questionnaires have been programmed in JavaScript using jsPsych [42], a JavaScript library for running behavioural experiments in a web browser that allows for accurate response time measurements [43]. Only participants can access the assessments, using a personal ID number. Once the assessments are completed, summary measures are created and temporarily stored against participants' personal IDs in a secure database before being transferred to a second, secure research database, against private ID numbers to ensure anonymisation.

(a) Cognitive measures Cognitive performance is being assessed with tests of processing speed, visual relational memory and core executive functions (working memory, inhibition and cognitive flexibility) [44] that are well 
validated in children and have been shown to be sensitive to the effects of PA $[11,45]$. The cognitive battery takes approximately $50 \mathrm{~min}$ to complete and comprises the following tasks:

1. Processing speed is assessed using a simple reaction time task.

2. Visual relational memory performance is assessed with a modified version of a previously described paradigm [10].

3. Inhibitory control is assessed with a modified version of the flanker task [46].

4. Working memory is assessed using a modified version of the visual 2-back task [47, 48].

5. Cognitive flexibility is assessed using a modified version of the colour-shape switch task [46].

Details of each task are provided in Additional file 2.

The task order is pseudo-randomised across participants within schools: all participants start with the reaction time task (i.e. processing speed), followed by the remaining four tasks in a random order (see Additional file 2 for details on the randomisation process). Teachers were instructed to ask pupils to complete the tasks at home, as pilot tests in three schools indicated that the local IT environment (e.g. bandwidth) may not be able to cope with large groups of pupils ( $>20$ ) completing the tasks at the same time, and it was deemed a logistical burden on schools to set the tasks during school lessons. Nevertheless, some schools have decided to complete the tasks during school time, as they felt this was easier to manage.

(b) Mental and physical health measures The mental and physical health questionnaire takes approximately 20 min to complete and is intentionally kept brief so that it can be easily completed in school. The questionnaire comprises the following core measures:

1. Mental health is assessed with the Strengths and Difficulties Questionnaire (SDQ) [49].

2. Global self-esteem is measured using the global selfesteem scale of the short version of the Physical Self-Description Questionnaire (P-SDQ) [50].

3. Physical self-esteem is measured using the physical self-esteem scale of the short version of the P-SDQ [50].

4. Global health is measured using a single item on a 5-point scale drawn from the Health Behaviour in School-Aged Children (HBSC) survey [24]: 'In general, how would you say your health is?' ( 5 = excellent to $1=$ poor).

5. Physical activity is measured with a single item [51]: 'In the past week, on how many days have you done a total of 60 minutes or more of physical activity, which was enough to raise your breathing rate? This may include sport, exercise, and brisk walking or cycling for fun, or to get to and from places'.

6. Habitual physical activity over the past 6 months is measured with a single item drawn from previous studies [52]: 'Thinking about the past six months, how often have you been physically active for an hour every day during a typical week in school term?' ( 1 = never to 7 = always).

We additionally explore the effect of the intervention on daytime sleepiness, PE enjoyment and psychological variables linked to daily MVPA.

7. Daytime sleepiness. During adolescence the circadian system delays and lengthens, causing sleep timing to shift later [53], which can lead to daytime sleepiness [54] and behavioural issues including school lateness, absenteeism and, ultimately, poor academic performance [55-57]. Based on evidence of a positive relationship between VPA and sleep quality and quantity in young adults [58] and suggestions that exercise programmes might improve aspects of adolescent sleep [59,60], the study will investigate the intervention's impact on daytime sleepiness using the Paediatric Daytime Sleepiness Scale (PDSS) [61].

8. PE enjoyment. To investigate the acceptability of the intervention with Year 8 pupils, we measure PE enjoyment with a single item [62]: 'I enjoy PE.' $(1=$ strongly disagree to $7=$ strongly agree $)$.

9. Psychological variables linked with daily MVPA. Understanding the extent to which a programme of VPA during PE might impact psychological variables with potential to explain variance in overall daily MVPA is important, given the low adolescent activity levels [24]. We use the Theory of Planned Behaviour $[63,64]$ and the Prototype Willingness Model [65] to investigate whether the intervention changes attitudes and beliefs about daily MVPA and active types of people. All items relating to constructs from the Theory of Planned Behaviour and the Prototype Willingness Model were developed according to established theoretical $[63,64]$ and practical $[66,67]$ guidelines. An overview of the measures is provided in Additional file 2.

Physical activity during PE Objective measures of VPA during $\mathrm{PE}$ are being collected in each school once during regular lessons at baseline (May-July 2017, and at some remaining schools in September 2017) and again during lessons in spring term (March-May 2018). Each school is visited at each timepoint to test at least $50 \%$ of the 
pupils in the year group. Measures are taken using the Axivity AX3, a wrist-worn tri-axial accelerometer designed by Open Lab, Newcastle University, UK. Trained research assistants show pupils how to fit the AX3 on their non-dominant wrist. The devices are attached after pupils change for PE and collected before they change back into school clothes. Class average minutes of MPA and VPA during active lesson time are calculated using established cut-points [68] and standardised, for comparison purposes, to minutes per hour.

Covariates Information about pupils' ethnicity and language proficiency is collected as part of the online assessments.

Brain imaging sub-study A sub-sample of participants is being recruited to participate in additional testing to investigate the effect of the physical activity intervention on brain structure and function.

The brain imaging sub-study runs in parallel with the main trial, making optimal use of the intervention. Assessments will take place pre-intervention (baseline), immediately post-intervention (t1) and 12 months postintervention (t2). The brain imaging sub-study consists of two sub-samples, recruited at different times during the trial:

1. The first cohort of 60 participants completed brain-study assessments pre-intervention, and will complete identical assessments post-intervention to investigate pre-to-post intervention changes. Participants will also be invited to complete a set of assessments 1 year following the intervention.

2. A second cohort of 50 participants will be recruited for post-intervention assessments that are similar, but not identical, to the assessments of the first cohort, as well as 1-year follow-up assessments. The addition of this cohort will enable cross-sectional analysis of between-group differences in a second sample.

Figure 3 shows a diagram of the participant flow, for each cohort, through the study. Details of recruitment for the brain imaging sub-study as well as an overview of assessments per cohort are provided in Additional file 2.

The sub-study has been granted separate ethical approval by the University of Oxford Medical Sciences Inter-Divisional Research Ethics Committee (Registration No. R51313) and was retrospectively registered (19 July 2018) at ClinicalTrials.gov (ID NCT03593863).

\section{(a) Brain imaging sub-study outcome measures}

1. Magnetic resonance imaging (MRI)
Scanning is carried out at the Wellcome Centre for Integrative Neuroimaging, Oxford, using a 3T Siemens Magnetom Prisma (Erlangen, Germany) scanner with a 32-channel head coil. The MRI protocol comprises both functional and structural sequences, takes approximately $55 \mathrm{~min}$ to complete and includes (1) T1-weighted (T1w) structural MRI, (2) resting-state functional MRI (rs-fMRI), (3) diffusion-weighted MRI (DW-MRI), (4) quantitative fast low angle shot (FLASH) MRI, (5) pseudo-continuous arterial spin labelling (pCASL) and (6) MR angiography (MRA; at post-intervention only). Full details of the MRI protocol are provided in Additional file 2.

\section{Cognitive measures}

Cognitive performance is assessed using tests of visual short-term memory (object-location task [69-71]), relational memory (relational memory task [10]), cognitive flexibility (colour-shape switch task [46]) and planning (Tower of London task [72-74]). Details of the cognitive assessments are provided in Additional file 2. The tests are completed in the lab at baseline (cohort 1), postintervention (cohorts 1 and 2) and follow-up (cohorts 1 and 2). Cohort 1 completes tests of visual short-term memory and mental associations as well as relational memory and cognitive flexibility. The latter tasks are taken from the main trial and repeated in the brain imaging sub-study to (a) obtain a well-controlled, labbased measure of the task for cross-validation, and (b) ensure that all brain-study participants will have a score on this task, independent from their main trial completion. Cohort 2 completes tests of visual shortterm memory and planning.

\section{Mental and physical health measures}

A comprehensive set of online questionnaires is completed by all participants at baseline (cohort 1), post-intervention (cohorts 1 and 2) and follow-up (cohorts 1 and 2).

(a) Attention-deficit hyperactivity disorder (ADHD) symptoms are assessed by the ADHD rating scale IV [75], completed by a parent of the participant.

(b) Puberty status is assessed using the Pubertal Development Rating Scale [76], a self-report measure of physical development for youths under the age of 16 .

(c) Mood is assessed using the abbreviated Profile of Mood States (POMS) [77]. The abbreviated POMS scale is a well-validated questionnaire that contains 40 self-report items on a 5 -point Likert scale. 


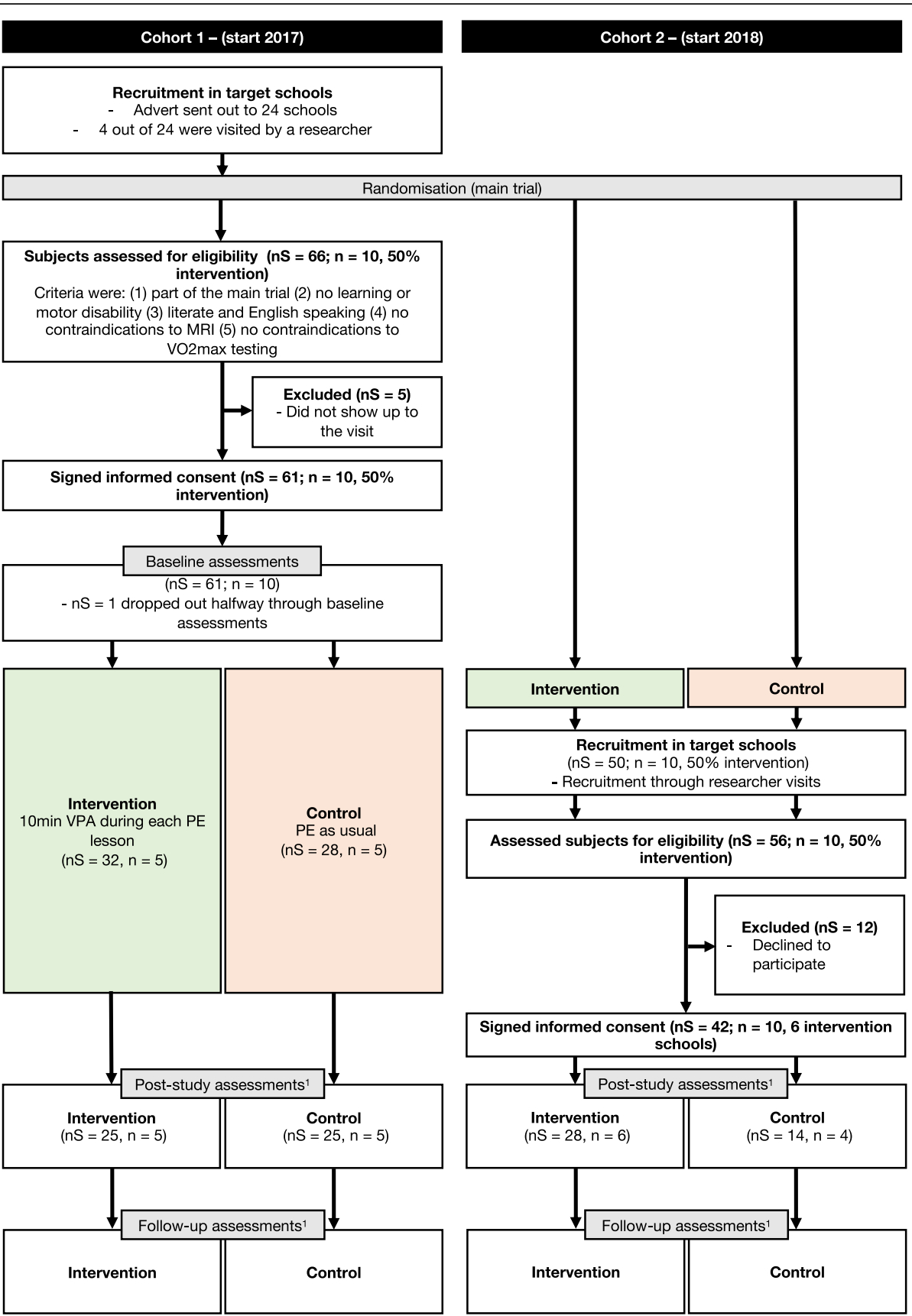

Fig. 3 Flow diagram brain imaging sub-study. ${ }^{1}$ Note: post-intervention assessments differ between cohorts 1 and 2 , but not within a cohort; see text and Additional file 2: Figure S7. Abbreviations: MRI magnetic resonance imaging, $n$ number of schools, nS number of subjects, $P E$ physical education, $\mathrm{VO}_{2}$ max maximal oxygen consumption, VPA vigorous physical activity

(d) Sleep is assessed using the Cleveland Adolescent Sleepiness Questionnaire (CASQ [78] and the Sleep Condition Indicator (SCI) [79]. The CASQ is a selfreport scale to measure excessive daytime sleepiness. The SCI is an 8-item self-report measure of insomnia symptoms, based on Diagnostic and Statistical Manual of Mental Disorders, $5^{\text {th }}$ edition
(DSM-5) criteria, and it captures both sleep problems and daytime functions.

(e) Mental health is assessed with the SDQ [49].

(f) General health and behaviour are assessed using various items of the HBSC survey (2009/2010) [80], capturing body image, eating behaviour, oral health, physical activity, leisure time activity, substance use, 
family structure, peer relationships, self-rated health and socio-economic environment.

\section{Psychological variables linked with daily MVPA.}

The study also includes exploratory explicit and implicit measures of psychological variables linked to daily average PA, using the frameworks set out in the Theory of Planned Behaviour $[63,64]$ and the Prototype Willingness Model [65]. Additional details are provided in Additional file 2.

5. Physical activity, fitness and gait (a) Physical activity

Daily average MVPA during a typical week in school term is measured using the Axivity AX3 accelerometer. A 7-day protocol (5 weekdays and 2 weekend days) is sufficient to obtain a reliability coefficient of 0.8 or above when assessing adolescent PA [81].

\section{(b) Cardiorespiratory fitness}

Cardiorespiratory fitness is measured using a standard maximal oxygen consumption $\left(\mathrm{VO}_{2} \mathrm{max}\right)$ incremental step test, in which the rate of oxygen consumption is measured while the participant cycles on an ergometer. Resistance is increased every minute until the participant reaches volitional exhaustion or is unable to maintain a cadence of 60 revolutions per minute (rpm). Additional details are provided in Additional file 2.

\section{(c) Gait}

Participants walk over a 10-m obstacle-free and flat surface walkway, while wearing a single inertial measurement unit (IMU, LPMS-B, Life Performance Research, Tokyo, Japan), to obtain temporal and spatial gait parameters [82, 83]. Participants perform two consecutive 10 -m walks, taking approximately $20 \mathrm{~s}$ to complete.

\section{Sample size/power calculation}

The primary intention-to-treat analysis will compare maths test performance between intervention and control schools, for which NatCen has performed a power calculation [36]. The initial power calculation, which demonstrated a range of minimum detectable effect sizes (MDESs) for various achieved samples [36], has been updated (in FebruaryMarch 2018) to reflect school drop-out ( $n=18$ schools) and a revised set of assumptions [37]. The newly computed MDES is equal to 0.24 standard deviations. The MDES would have been 0.21 standard deviations if all 106 recruited schools had participated in the trial. Power calculations were performed in PowerUp! [84].

\section{Data analysis plan}

In accordance with the Consolidated Standards of Reporting Trials (CONSORT) 2010 statement for cluster-randomised controlled trials guidelines [85], the cluster and participant flow will be reported. Descriptive statistics of recruitment and drop-out will be provided on both the cluster and subject levels. Further, completeness of, and adherence to, the intervention will be reported on a cluster-level only. Baseline characteristics of the randomised groups will be summarised across schools and/or pupils, and will be presented on a pupil level where appropriate.

The primary intention-to-treat analysis comparing maths test performance between intervention and control schools will be conducted by NatCen and is described elsewhere [37]. To account for differences between pupils at baseline, baseline maths performance (Key Stage 2 test score) will be collected from the National Pupil Database (NPD). Analysis of secondary outcome measures will be conducted on an intention-to-treat basis. Per-protocol analyses of secondary measures will also be reported.

Multilevel modelling will be used, as these analyses take the hierarchical structure of the data into account (i.e. pupils within schools) [86]. The models will be used to assess the effect of the intervention, as well as to explore associations between secondary outcome measures, and will adjust for various confounding factors (e.g. sex, socio-economic status, school gender status). Structural equation models (SEMs) may be used for mediation analyses.

MRI data collected as part of the brain imaging substudy will be analysed and processed using tools from MrTrix3, MATLAB, the FMRIB Software Library (FSL), statistical parametric mapping (SPM) and FreeSurfer, employing parametric and non-parametric statistical analysis methods where appropriate. Details of data (pre) processing and analysis are provided in Additional file 2.

\section{Discussion}

The primary aim of the Fit to Study trial is to investigate the impact of a one academic year (10 months) VPA programme delivered during $\mathrm{PE}$ on academic performance in Year 8 pupils. This protocol describes the secondary aims of the trial, including the effect of the intervention on cardiorespiratory fitness, cognitive performance, mental health and the brain. The findings of this trial will contribute to the growing evidence base of the effects of PA on academic achievement $[9,19,87,88]$ and will advance our understanding of the effect of PA on a range of variables - fitness, cognition, mental health and the brain that are thought to moderate or mediate this relationship. Although many of these variables have individually been linked to PA [4, 11, 60, 89], their role in PA-academic achievement relationships is still unclear. 
The early years of secondary school are a period of dynamic neurobiological change. Evidence that additional VPA during school PE has a positive impact on the brain, cognitive function and attainment has the potential to help policymakers and educators steer the developmental course in a positive direction. The Fit to Study intervention could offer a feasible method of increasing VPA without disrupting the curriculum. Further potential advantages are its scalability and ease of dissemination, which are important if the intervention is to be used more widely in the future [90]. The intervention may be incorporated in PE lessons without taking up additional PE time; it should not interfere with the curriculum and does not require extensive training of teachers.

The results could provide further evidence that school is an effective setting for increasing adolescent PA and positively influencing their PA behaviour [91] irrespective of their parents' PA behaviour or socio-economic status. This is important, given that pupils from deprived backgrounds often have lower PA levels [92-94] and are more difficult to reach via non-school interventions. The findings could also add to evidence on whether PA interventions during $\mathrm{PE}$, including those that supplement $\mathrm{PE}$ lessons with vigorous $\mathrm{PA}$, are effective at increasing PA levels [95, 96] and improving cognition [11].

The findings of the Fit to Study trial will provide valuable information for other research groups and organisations looking to design and implement school-based PA interventions. In addition, the trial has the potential to inform the political and scientific debate regarding the role of schools in promoting PA among adolescents.

\section{Dissemination}

The findings of this trial will be published in peer-reviewed journals, irrespective of the direction or magnitude of the results, and will also be presented at national and international scientific meetings. If permitted by journal policies, the results will be made available online wherever possible.

\section{Trial status}

Recruitment of schools for the main trial is complete, the intervention is being delivered and data collection for secondary measures is underway. Recruitment of participants for the brain study is underway.

The trial sponsor is the University of Oxford. The Protocol version is 1 .

\section{Additional files}

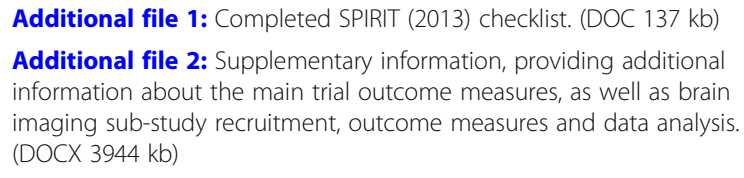

\section{Abbreviations}

ADHD: Attention-deficit hyperactivity disorder; CASQ: Cleveland Adolescent Sleepiness Questionnaire; CONSORT: Consolidated Standards of Reporting Trials; DW-MRI: Diffusion-weighted magnetic resonance imaging; FLASH: Fast low angle shot; FSL: FMRIB Software Library; FSM: Free school meals; HBSC: Health Behaviour in School-aged Children; HR: Heart rate; MDES: Minimum detectable effect size; MRA: Magnetic resonance Angiography; MRI: Magnetic resonance imaging; MVPA: Moderate-tovigorous physical activity; NFER: National Foundation for Educational Research; NPD: National Pupil Database; PA: Physical activity; PCASL: Pseudocontinuous arterial spin labelling; PDSS: Paediatric Daytime Sleepiness Scale; PE: Physical education; POMS: Profile of Mood States; P-SDQ: Physical SelfDescription Questionnaire; PTM: Progress Test in Maths; RCT: Randomised controlled trial; rpm: Revolutions per minute; rs-fMRI: Resting-state functional magnetic resonance imaging; SCl: Sleep Condition Indicator; SDQ: Strengths and Difficulties Questionnaire; SPIRIT: Standard Protocol Items: Recommendations for Interventional Trials; SPM: Statistical parametric mapping; T1w: T1-weighted; $\mathrm{VO}_{2}$ max: Maximal oxygen consumption; VPA: Vigorous physical activity

\section{Acknowledgements}

We are grateful to Emily Curtis, Emma Eldridge, Emily Plester, Eneid Leika, Oliver Bushnell, Cyrus Goodger and Tom Smejka for their help with data collection, as well as to Kate Curtis and Richard Neil for their advice on intervention development and testing in schools. We would also like to thank Thomas (Tom) Okell, Daniel Papp, Jon Campbell, Michael Sanders, Cristina Malamateniou and Nils Forkert for their advice on the MRI protocol, as well as the FMRIB radiographers for their help with scanning the participants of the brain imaging sub-study. We would like to thank Patrick Esser for his input on actigraphy analysis. We would like to thank Josh de Leeuw for assistance with programming in jsPsych. Finally, we would like to thank all the schools, teachers and pupils taking part in Fit to Study.

\section{Funding}

The research is funded by the Education Endowment Foundation (EEF) and the Wellcome Trust under their Education and Neuroscience Programme (Grant Reference 2681). The Wellcome Centre for Integrative Neuroimaging is supported by core funding from the Wellcome Trust (203139/Z/16/Z). HJB is a Wellcome Trust Principal Research Fellow (110027/Z/15/Z).

\section{Availability of data and materials}

The main trial datasets generated and/or analysed during the current study are not publicly available while the study is underway due to the sensitivity of the data, but are available from the corresponding author on reasonable request. Once the trial is complete, the anonymised data will be stored in the Education Endowment Foundation's archive form in the UK Data Archive.

The Key Stage 2 data that support the findings of this study are available from the National Pupil Database, but restrictions apply to the availability of these data, which are used under license for the current study, and so are not publicly available. Data will however be available from the authors upon reasonable request and with permission of the National Pupil Database. The datasets generated and/or analysed as part of the brain imaging sub-study will be available from the corresponding author on reasonable request.

\section{Authors' contributions}

$H J B$ and $H D$ are the principal investigators and conceived the study. NB is the trial coordinator. TW, CW, PS, NB, AM, JP, KA, JD, HD and HJB contributed to the design of the study. TW, CW, NB and PS are responsible for data collection. TW and CW drafted the manuscript. All authors provided comments and approved the final manuscript.

\section{Ethics approval and consent to participate}

All schools were required to read and sign a memorandum of understanding. Participants and their parent(s)/guardian(s) were asked to read the information sheet and provide opt-out consent. Schools consented for their anonymised data to be published. Individuals who did not opt out of data collection and storage agreed for their anonymised data to be published. The trial has been granted ethical approval by the Central University Research Ethics Committee of Oxford University (Registration No. R48879/RE001). The trial's protocol was retrospectively registered at ClinicalTrials.gov on 18 September 2017 (NCT03286725). Participants in the brain study and their parent(s)/guardian(s) 
were required to read the information sheet and consent to participate in this part of the trial. The brain study, embedded in the trial, has been granted separate ethical approval by University of Oxford Medical Sciences InterDivisional Research Ethics Committee (Registration No. R51313). The brain imaging sub-study protocol was retrospectively registered at ClinicalTrials.gov on 19 July 2018 (NCT03593863)

\section{Consent for publication}

\section{Not applicable.}

\section{Competing interests}

The authors declare that they have no competing interests.

\section{Publisher's Note}

Springer Nature remains neutral with regard to jurisdictional claims in published maps and institutional affiliations.

\section{Author details}

${ }^{1}$ Wellcome Centre for Integrative Neuroimaging, FMRIB Centre, Nuffield Department of Clinical Neurosciences, University of Oxford, John Radcliffe Hospital, Headley Way, Oxford OX3 9DU, UK. ² Centre for Movement, Occupational and Rehabilitation Sciences (MOReS), Oxford Brookes University, Headington Campus, Oxford OX3 OBP, UK. ${ }^{3}$ School of Sport, Exercise and Rehabilitation Sciences, University of Birmingham, Birmingham B15 2TT, UK

Received: 30 July 2018 Accepted: 4 March 2019

Published online: 02 April 2019

\section{References}

1. Foulkes L, Blakemore S-J. Studying individual differences in human adolescent brain development. Nat Neurosci. 2018;21:315-23.

2. Sawyer SM, Afifi RA, Bearinger LH, Blakemore SJ, Dick B, Ezeh AC, et al. Adolescence: a foundation for future health. Lancet. 2012;379:1630-40.

3. Dahl RE, Allen NB, Wilbrecht $L$, Suleiman AB. Importance of investing in adolescence from a developmental science perspective. Nature. 2018;554:441.

4. Gomez-Pinilla F, Hillman $\mathrm{CH}$. The influence of exercise on cognitive abilities. Compr Physiol. 2013;3:403-28.

5. Voss MW, Vivar C, Kramer AF, van Praag H. Bridging animal and human models of exercise-induced brain plasticity. Trends Cogn Sci. 2013;17:525-44

6. Cotman CW, Berchtold NC, Christie L-A. Exercise builds brain health: key roles of growth factor cascades and inflammation. Trends Neurosci. 2007:30: 464-72.

7. Hillman $\mathrm{CH}$, Erickson $\mathrm{Kl}$, Kramer AF. Be smart, exercise your heart: exercise effects on brain and cognition. Nat Rev Neurosci. 2008;9:58-65.

8. Hillman $\mathrm{CH}$, Erickson Kl, Hatfield BD. Run for your life! Childhood physical activity effects on brain and cognition. Kinesiol Rev. 2017;6:12-21.

9. Donnelly JE, Hillman CH, Castelli D, Etnier JL, Lee S, Tomporowski P, et al. Physical activity, fitness, cognitive function, and academic achievement in children: a systematic review. Med Sci Sports Exerc. 2016:48:1197.

10. Chaddock L, Erickson Kl, Prakash RS, Kim JS, Voss MW, Vanpatter M, et al. A neuroimaging investigation of the association between aerobic fitness, hippocampal volume, and memory performance in preadolescent children. Brain Res. 2010;1358:172-83.

11. Álvarez-Bueno C, Pesce C, Cavero-Redondo I, Sánchez-López M, MartínezHortelano JA, Martínez-Vizcaíno V. The effect of physical activity interventions on children's cognition and metacognition: a systematic review and metaanalysis. J Am Acad Child Adolesc Psychiatry. 2017;56:729-38.

12. de Greeff JW, Bosker RJ, Oosterlaan J, Visscher C, Hartman E. Effects of physical activity on executive functions, attention and academic performance in preadolescent children: a meta-analysis. J Sci Med Sport. 2018;21:501-7

13. Vazou S, Pesce C, Lakes K, Smiley-Oyen A. More than one road leads to Rome: a narrative review and meta-analysis of physical activity intervention effects on cognition in youth. Int J Sport Exerc Psychol. 2016;0:1-26.

14. Moreau D, Kirk IJ, Waldie KE. High-intensity training enhances executive function in children in a randomized, placebo-controlled trial. Elife. 2017;6:e25062.

15. Hsieh S, Lin C, Chang Y, Huang C, Hung T, City T, et al. Effects of childhood gymnastics program on spatial working memory. Med Sci Sports Exerc. 2017;49:2537-47.
16. Lind RR, Geertsen SS, Ørntoft C, Larsen MN, Dvorak J, Ritz C, et al. Improved cognitive performance in preadolescent Danish children after the schoolbased physical activity programme "FIFA 11 for Health" for Europe - a cluster-randomised controlled trial. Eur J Sport Sci. 2018;18:130-9.

17. Tomporowski PD, McCullick B, Pendleton DM, Pesce C. Exercise and children's cognition: the role of exercise characteristics and a place for metacognition. J Sport Heal Sci. 2015;4:47-55.

18. Booth JN, Leary SD, Joinson C, Ness AR, Tomporowski PD, Boyle JM, et al. Associations between objectively measured physical activity and academic attainment in adolescents from a UK cohort. Br J Sports Med. 2014;48:265-70.

19. Álvarez-Bueno C, Pesce C, Cavero-Redondo I, Sánchez-López M, GarridoMiguel M, Martínez-Vizcaíno V. Academic achievement and physical activity: a meta-analysis. Pediatrics. 2017;140:e20171498.

20. Lees C, Hopkins J. Effect of aerobic exercise on cognition, academic achievement, and psychosocial function in children: a systematic review of randomized control trials. Prev Chronic Dis. 2013;10:130010.

21. World Health Organization. Global recommendations on physical activity for health. Geneva: World Health Organization; 2010.

22. McMahon EM, Corcoran P, O'Regan G, Keeley H, Cannon M, Carli V, et al. Physical activity in European adolescents and associations with anxiety, depression and well-being. Eur Child Adolesc Psychiatry. 2017;26:111-22.

23. Sallis JF, Bull F, Guthold R, Heath GW, Inoue S, Kelly P, et al. Progress in physical activity over the Olympic quadrennium. Lancet. 2016;388:1325-36.

24. Inchley J et al. eds. Growing up unequal: gender and socioeconomic differences in young people's health and well-being. Health Behaviour in School-aged Children (HBSC) study: international report from the 2013/2014 survey. Copenhagen: WHO Regional Office for Europe; 2016 (Health Policy for Children and Adolescents, No. 7).

25. Paus T, Keshavan M, Giedd JN. Why do many psychiatric disorders emerge during adolescence? Nat Rev Neurosci. 2008;9:947.

26. Harter S. Causes and consequences of low self-esteem in children and adolescents. In: Baumeister RF, editor. Self-esteem. The Plenum series in social/clinical psychology. Boston: Springer; 1993. p. 87-116.

27. Veldman K, Bültmann U, Stewart RE, Ormel J, Verhulst FC, Reijneveld SA. Mental health problems and educational attainment in adolescence: 9-year follow-up of the TRAILS study. PLOS One. 2014;9:e101751.

28. Deighton J, Humphrey N, Belsky J, Boehnke J, Vostanis P, Patalay P. Longitudinal pathways between mental health difficulties and academic performance during middle childhood and early adolescence. $\mathrm{Br} J \mathrm{Dev}$ Psychol. 2018;36:110-26.

29. Kriemler S, Meyer U, Martin E, van Sluijs EMF, Andersen LB, Martin BW. Effect of school-based interventions on physical activity and fitness in children and adolescents: a review of reviews and systematic update. Br J Sports Med. 2011;45:923-30.

30. Hollis JL, Sutherland R, Williams AJ, Campbell E, Nathan N, Wolfenden L, et al. A systematic review and meta-analysis of moderate-to-vigorous physical activity levels in secondary school physical education lessons. Int J Behav Nutr Phys Act. 2017;14:52.

31. Fairclough S, Stratton G. Physical activity levels in middle and high school physical education: a review. Pediatr Exerc Sci. 2005;17:217.

32. Buchheit $M$, Laursen PB. High-intensity interval training, solutions to the programming puzzle: Part I: cardiopulmonary emphasis. Sport Med. 2013:43:313-38.

33. Costigan SA, Eather N, Plotnikoff RC, Taaffe DR, Lubans DR. Highintensity interval training for improving health-related fitness in adolescents: a systematic review and meta-analysis. Br J Sports Med. 2015;49:1253-61.

34. Costigan SA, Eather N, Plotnikoff RC, Hillman CH, Lubans DR. High-intensity interval training for cognitive and mental health in adolescents. Med Sci Sports Exerc. 2016:48:1985-93.

35. Logan GRM, Harris N, Duncan S, Schofield G. A review of adolescent highintensity interval training. Sport Med. 2014;44:1071-85.

36. Husain F. Fit to study - evaluation protocol. 2016. Available from: https:// educationendowmentfoundation.org.uk/public/files/Projects/Evaluation Protocols/Neuroscience_-_Fit_to_Study_Trial_Protocol.pdf

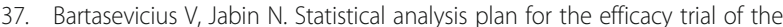
Fit to Study intervention. 2018. Available from: https:// educationendowmentfoundation.org.uk/public/files/Projects/Fit_to_Study_ SAP_2018.03.15_FINAL.pdf

38. GL Assessment. Progress test in Maths, level 13. Available from: https://www.glassessment.co.uk/products/progress-test-in-maths-ptm/. Accessed 27 Mar 2018. 
39. Léger LA, Mercier D, Gadoury C, Lambert J. The multistage 20 metre shuttle run test for aerobic fitness. J Sports Sci. 1988;6:93-101.

40. Mayorga-Vega D, Aguilar-Soto P, Viciana J. Criterion-related validity of the 20-M shuttle run test for estimating cardiorespiratory fitness: a metaanalysis. J Sport Sci Med. 2015;14:536-47.

41. Cooper KH. A means of assessing maximal oxygen intake. JAMA. 1968; 203:201-4.

42. de Leeuw JR. jsPsych: a JavaScript library for creating behavioral experiments in a Web browser. Behav Res Methods. 2015;47:1-12.

43. de Leeuw JR, Motz BA. Psychophysics in a web browser? Comparing response times collected with JavaScript and Psychophysics toolbox in a visual search task. Behav Res Methods. 2016;48:1-12.

44. Diamond A. Executive functions. Annu Rev Psychol. 2013;64:135-68.

45. Chang YK, Labban JD, Gapin Jl, Etnier JL. The effects of acute exercise on cognitive performance: a meta-analysis. Brain Res. 2012;1453:87-101.

46. Hillman CH, Pontifex MB, Castelli DM, Khan NA, Raine LB, Scudder MR, et al. Effects of the FITKids randomized controlled trial on executive control and brain function. Pediatrics. 2014;134:e1063-71.

47. Chen A-G, Yan J, Yin H-C, Pan C-Y, Chang Y-K. Effects of acute aerobic exercise on multiple aspects of executive function in preadolescent children. Psychol Sport Exerc. 2014;15:627-36.

48. Guiney $H$, Machado L. Benefits of regular aerobic exercise for executive functioning in healthy populations. Psychon Bull Rev. 2013;20:73-86.

49. Goodman R. The Strengths and Difficulties Questionnaire: a research note. J Child Psychol Psychiatry. 1997;38:581-6.

50. Marsh HW, Martin AJ, Jackson S. Introducing a short version of the physical self description questionnaire: new strategies, short-form evaluative criteria, and applications of factor analyses. J Sport Exerc Psychol. 2010;32:438-82.

51. Scott JJ, Morgan PJ, Plotnikoff RC, Lubans DR. Reliability and validity of a single-item physical activity measure for adolescents. J Paediatr Child Health. 2015:51:787-93.

52. Hagger MS, Chatzisarantis N, Biddle SJH, Orbell S. Antecedents of children's physical activity intentions and behaviour: predictive validity and longitudinal effects. Psychol Health. 2001;16:391-407.

53. Crowley SJ, Acebo C, Carskadon MA. Sleep, circadian rhythms, and delayed phase in adolescence. Sleep Med. 2007;8:602-12.

54. Gradisar M, Gardner G, Dohnt H. Recent worldwide sleep patterns and problems during adolescence: a review and meta-analysis of age, region, and sleep. Sleep Med. 2011:12:110-8.

55. Beebe DW. Cognitive, behavioral, and functional consequences of inadequate sleep in children and adolescents. Pediatr Clin. 2011:58:649-65.

56. Dahl RE, Lewin DS. Pathways to adolescent health sleep regulation and behavior. J Adolesc Health. 2002;31:175-84.

57. Dewald JF, Meijer AM, Oort FJ, Kerkhof GA, Bögels SM. The influence of sleep quality, sleep duration and sleepiness on school performance in children and adolescents: a meta-analytic review. Sleep Med Rev. 2010;14:179-89.

58. Gerber M, Brand S, Herrmann C, Colledge F, Holsboer-Trachsler E, Pühse U. Increased objectively assessed vigorous-intensity exercise is associated with reduced stress, increased mental health and good objective and subjective sleep in young adults. Physiol Behav. 2014;135:17-24.

59. Dolezal BA, Neufeld EV, Boland DM, Martin JL, Cooper CB. Interrelationship between sleep and exercise: a systematic review. Adv Prev Med. 2017;2017. https://doi.org/10.1155/2017/1364387.

60. Mendelson M, Borowik A, Michallet A, Perrin C, Monneret D, Faure P, et al. Sleep quality, sleep duration and physical activity in obese adolescents: effects of exercise training. Pediatr Obes. 2016;11:26-32.

61. Drake C, Nickel C, Burduvali E, Roth T, Jefferson C, Badia P, et al. The pediatric daytime sleepiness scale (PDSS): sleep habits and school outcomes in middle-school children. Sleep. 2003;26:455-8.

62. Prochaska JJ, Sallis JF, Slymen DJ, McKenzie TL. A longitudinal study of children's enjoyment of physical education. Pediatr Exerc Sci. 2003;15:170-8.

63. Ajzen I. From intentions to actions: a theory of planned behavior. Berlin: Springer; 1985.

64. Ajzen I. The theory of planned behavior. Organ Behav Hum Decis Process. 1991:50:179-211.

65. Gerrard M, Gibbons FX, Houlihan AE, Stock ML, Pomery EA. A dual-process approach to health risk decision making: the prototype willingness model. Dev Rev. 2008;28:29-61.

66. Fishbein $M$, Ajzen I. Predicting and changing behavior: the reasoned action approach. New York: Psychology Press; 2011.
67. Michie S, Johnston M, Francis J, Hardeman W, Eccles M. From theory to intervention: mapping theoretically derived behavioural determinants to behaviour change techniques. Appl Psychol. 2008;57:660-80.

68. Phillips LRS, Parfitt G, Rowlands AV. Calibration of the GENEA accelerometer for assessment of physical activity intensity in children. J Sci Med Sport. 2013;16:124-8.

69. Pertzov Y, Dong MY, Peich MC, Husain M. Forgetting what was where: the fragility of object-location binding. PLOS One. 2012;7:e48214.

70. Liang Y, Pertzov Y, Nicholas JM, Henley SMD, Crutch S, Woodward F, et al. Visual short-term memory binding deficit in familial Alzheimer's disease. Cortex. 2016;78:150-64.

71. Pertzov Y, Miller TD, Gorgoraptis N, Caine D, Schott JM, Butler C, et al. Binding deficits in memory following medial temporal lobe damage in patients with voltage-gated potassium channel complex antibodyassociated limbic encephalitis. Brain. 2013:136:2474-85.

72. Shallice T. Specific impairments of planning. Philos Trans R Soc B Biol Sci. 1982:298:199-209.

73. Anderson P, Anderson V, Lajoie G. The Tower of London Test: validation and standardization for pediatric populations. Clin Neuropsychol. 1996;10:54-65.

74. van der Niet AG, Smith J, Scherder EJA, Oosterlaan J, Hartman E, Visscher C. Associations between daily physical activity and executive functioning in primary school-aged children. J Sci Med Sport. 2015;18:673-7.

75. DuPaul GJ, Power TJ, Anastopoulos AD, Reid R. ADHD rating scale-IV: checklists, norms, and clinical interpretation. New York: Guilford; 1998

76. Peterson A, Crockett L, Richards M, Boxer A. A self-report measure of pubertal status. J Youth Adolesc. 1988;17:117-33.

77. Grove JR, Prapavessis H. Preliminary evidence for the reliability and validity of an abbreviated profile of mood states. Int J Sport Psychol. 1992:23:93-109.

78. Spilsbury JC, Drotar D, Rosen CL, Redline S. The Cleveland Adolescent Sleepiness Questionnaire: a new measure to assess excessive daytime sleepiness in adolescents. J Clin Sleep Med. 2007;3:603-12.

79. Espie CA, Kyle SD, Hames P, Gardani M, Fleming L, Cape J. The sleep condition indicator: a clinical screening tool to evaluate insomnia disorder. BMJ Open. 2014:4:1-6.

80. Currie C, Griebler R, Inchley J, Theunissen A, Molcho M, Samdal O, et al. Health Behaviour in School-aged Children (HBSC) study protocol: background, methodology and mandatory items for the 2009/10 survey. Edinburgh and Vienna: CAHRU and LBIHPR; 2010.

81. Trost SG, Pate RR, Freedson PS, Sallis JF, Taylor WC. Using objective physical activity measures with youth: how many days of monitoring are needed? Med Sci Sports Exerc. 2000;32:426-31.

82. Esser P, Dawes H, Collett J, Howells K. IMU: inertial sensing of vertical CoM movement. J Biomech. 2009:42:1578-81.

83. Esser P, Dawes H, Collett J, Feltham MG, Howells K. Assessment of spatiotemporal gait parameters using inertial measurement units in neurological populations. Gait Posture. 2011;34:558-60.

84. Dong N, Maynard R. PowerUp!: a tool for calculating minimum detectable effect sizes and minimum required sample sizes for experimental and quasiexperimental design studies. J Res Educ Eff. 2013;6:24-67.

85. Campbell MK, Piaggio G, Elbourne DR, Altman DG. Consort 2010 statement: extension to cluster randomised trials. BMJ. 2012:345:1-21.

86. Campbell MK, Mollison J, Steen N, Grimshaw JM, Eccles M. Analysis of cluster randomized trials in primary care: a practical approach. Fam Pract. 2000;17:192-6.

87. Marques A, Gómez F, Martins J, Catunda R, Sarmento H. Association between physical education, school-based physical activity, and academic performance: a systematic review. Retos. 2017:31:316-20.

88. Sullivan RA, Kuzel $A M H$, Vaandering ME, Chen W. The association of physical activity and academic behavior: a systematic review. J Sch Health. 2017:87:388-98.

89. Biddle SJH, Asare M. Physical activity and mental health in children and adolescents: a review of reviews. Br J Sports Med. 2011:45:886-95.

90. Reis RS, Salvo D, Ogilvie D, Lambert EV, Goenka S, Brownson RC. Scaling up physical activity interventions worldwide: stepping up to larger and smarter approaches to get people moving. Lancet. 2016;388: 1337-48.

91. Hills AP, Dengel DR, Lubans DR. Supporting public health priorities: recommendations for physical education and physical activity promotion in schools. Prog Cardiovasc Dis. 2015;57:368-74. 
92. Richter M, Erhart M, Vereecken CA, Zambon A, Boyce W, Gabhainn SN. The role of behavioural factors in explaining socio-economic differences in adolescent health: a multilevel study in 33 countries. Soc Sci Med. 2009;69: 396-403.

93. Eime RM, Charity MJ, Harvey JT, Payne WR. Participation in sport and physical activity: Associations with socio-economic status and geographical remoteness. BMC Public Health. 2015;15:434.

94. Drenowatz C, Eisenmann J, Pfeiffer K, Heelan K, Gentile D, Walsh D. Influence of socio-economic status on habitual physical activity and sedentary behavior in 8- to 11-year old children. BMC Public Health. 2010;10:1-11.

95. Lonsdale C, Rosenkranz RR, Peralta LR, Bennie A, Fahey P, Lubans DR. A systematic review and meta-analysis of interventions designed to increase moderate-to-vigorous physical activity in school physical education lessons. Prev Med (Baltim). 2013;56:152-61.

96. 2018 Physical Activity Guidelines Advisory Committee. 2018 physical activity guidelines advisory committee scientific report. Washington, DC: Office of Disease Prevention and Health Promotion; 2018.

Ready to submit your research? Choose BMC and benefit from:

- fast, convenient online submission

- thorough peer review by experienced researchers in your field

- rapid publication on acceptance

- support for research data, including large and complex data types

- gold Open Access which fosters wider collaboration and increased citations

- maximum visibility for your research: over $100 \mathrm{M}$ website views per year

At $\mathrm{BMC}$, research is always in progress.

Learn more biomedcentral.com/submissions 